\title{
MODELS OF THE INTERACTION BETWEEN ELECTRONS AND POLAR OPTICAL PHONONS IN NANOSTRUCTURES
}

\author{
B.K. Ridley \\ Department of Computing Science and Electronic Engineering, University of Essex, Colchester CO4 3SQ, United Kingdom \\ E-mail: bkr@essex.ac.uk
}

Received 16 April 2015; accepted 29 September 2015

\begin{abstract}
A brief summary is made of the models used to describe the interaction between electrons and polar optical phonons in nanostructures. Simpler models are compared with the model that describes optical modes that satisfy both mechanical and electrical boundary conditions. Satisfaction of these boundary conditions requires modes to be a linear combination (LC) of longitudinal (LO), transverse (TO) and interface (IF) modes. The role of lattice dispersion turns out to be crucial. If accuracy is not essential, the simple models can provide adequate results, provided that coupled-mode and hot-phonon effects are absent.

Keywords: electron-phonon interactions, electronic transport, nanostructures
\end{abstract}

PACS: 72.10.Di, 73.63.-b

\section{General}

In the study of transport phenomena in semiconductor nanostructures the interaction between electrons and phonons assumes a central significance because of its intrinsic nature. In principle, scattering due to impurities, lattice defects and interface roughness can be eliminated. In practice, technical innovation regarding purification and crystal growth can make non-phonon scattering subordinate in many structures at room temperature, and likely to be dominant only at low temperatures, where the excitation of lattice vibrations is weak, but even there, the electron-phonon interaction cannot be ignored when the electrons become hot in high electric fields. The only other scattering mechanism that is not readily amenable to ideal crystal growth is that due to alloy fluctuations. Otherwise, in unalloyed binary semiconductors, the electron-phonon interaction is of central importance.

In nanostructures both electrons and phonons are confined, and a description of the electron-phonon interaction must necessarily contain models of confinement. The confinement of electrons is determined by the potential barrier at each interface of the structure. The boundary conditions that have to be satisfied are the continuity of the wavefunction and its gradient. In many cases, the condition affecting the gradient of the Bloch function, $u(\mathbf{r}) F(\mathbf{r})$, where $F(\mathbf{r})$ is an envelope function, is satisfactorily replaced by $\mathrm{m}^{\star-1} \mathrm{~d} F(z) / \mathrm{d} z$, where $\mathrm{m}^{\star}$ is the effective mass and $z$ is along the normal to the interface [1, 27. Or, more simply, in cases where the barrier is high, the boundary condition reduces to $F(z)=0$. The model of electron confinement, as outlined above, is uncontroversial.

The same cannot be said of the models of confinement of optical phonons. The boundary conditions affecting acoustic modes - continuity of particle displacement and of elastic stress - are the ones of classical physics and, again, uncontroversial. The boundary conditions for optical modes are more complex, and their elucidation is relatively recent [3, 印. Previous to this, three models were proposed. The first simply ignored optical phonon confinement entirely and calculated the electron-phonon interaction using bulk modes [5]. We label this the 3DP model. Surprisingly, the result proved to be close to the result for confined phonons. The second focused on the electric fields of the polar modes and redefined the medium as a dielectric continuum (DC). Following the treatment of the polar slab by Fuchs 
and Kliewer [6], the DC model takes the phonon spectrum to be confined longitudinal optical (LO) modes at the zone-centre frequency plus two interface (IF) modes, the frequency of one deriving from the well material, the other from the barrier(s), all satisfying the classical electrical boundary conditions - continuity of electric potential and of normal electric displacement. Again, surprisingly, the results proved to be close to those derived from more accurate models. However, the form of the mode patterns revealed by Raman scattering was not that of the DC model. Nor, obviously, could the DC model be applied to any non-polar system, such as $\mathrm{Si} / \mathrm{Ge}$. The third model ignored electrical boundary conditions and assumed that the relevant boundary conditions were those of acoustic modes, those also of hydrodynamics [7]. The hydrodynamics (HD) predicted exactly those observed by Raman scattering [8], and it was a model that was applicable to non-polar materials. The model assumed that the elastic stress for optical modes was of the same form as for acoustic modes, which proved to be true for non-polar material, but not for polar material. An analysis by Akero and Ando [9], based on the linear-chain model, showed that the correct boundary condition must include the variation of the ionic masses, which the classical boundary conditions for acoustic modes do not. It was clear that none of the three models was correct.

From the classical study of acoustic modes in a non-polar slab, it was found that the acoustic boundary conditions could not be satisfied for a single mode with components of particle displacement along the normal to the surface. For such cases it was necessary for there to be a coherent linear combination of longitudinal acoustic (LA) and transverse acoustic (TA) modes. It became clear that the satisfaction of both mechanical and electrical boundary conditions required a coherent linear combination (LC) of LO, TO and IF modes. But the form of the mechanical boundary condition involving stress remained unclear. Fortunately, this problem could be bypassed in many cases of interest by exploiting the large disparity of frequency and other properties between the barrier and well and assuming that the ionic displacement vanished at the interface. This allowed the correct eigenmodes to be established [10-13], and was shown to give results in close agreement with those obtained by computer intensive microscopic modeling [14]. An analytical microscopic model became available [3] that confirmed the dependence on mass shown by Akero and Ando and defined the mechanical boundary conditions for optical modes. The LC model with these mechanical boundary conditions along with the usual electrical boundary conditions is the most accurate analytical continuum model available.

\section{Dispersion}

Unlike other models, the LC model takes full account of lattice dispersion. In a long-wavelength approximation, it takes the frequency of the hybrid mode to be quadratic in the wave vector

$$
\omega^{2}=\omega_{\mathrm{L}}^{2}-v_{\mathrm{L}}^{2} q^{2}
$$

where $\omega_{\mathrm{L}}$ is the zone-centre frequency and $v_{\mathrm{L}}$ is the velocity. In the simplest model, $v_{\mathrm{L}}$ is just the velocity of the LA mode. Dispersion has important consequences. The first is the effect on the dielectric functions associated with the reduction of frequency with the wave vector. For the LO component, the permittivity becomes

$$
\varepsilon(\omega)=\varepsilon_{\infty} \frac{\omega^{2}-\omega_{\mathrm{L}}^{2}+v_{\mathrm{L}}^{2} q^{2}}{\omega^{2}-\omega_{\mathrm{T}}^{2}+v_{\mathrm{L}}^{2} q^{2}}
$$

where $\omega_{\mathrm{T}}$ is the zone-centre TO frequency. This means that for the LO component $\varepsilon(\omega)=0$ for all wavevectors. The fundamental dispersion for the IF component is electromagnetic, which makes $q_{z}^{2}=-$ $q_{x}^{2}$ in the unretarded limit. However, as a coherent component of the LC model, it must have the frequency of the LO component. Thus

$$
\varepsilon(\omega)=\varepsilon_{\infty} \frac{\omega^{2}-\omega_{\mathrm{L}}^{2}}{\omega^{2}-\omega_{\mathrm{T}}^{2}}=-\varepsilon_{\infty} \frac{\nu_{\mathrm{L}}^{2} q^{2}}{\omega_{\mathrm{L}}^{2}-\omega_{\mathrm{T}}^{2}-v_{\mathrm{L}}^{2} q^{2}} .
$$

Moreover, the electric field associated with the IF component and its ionic displacement, $\mathbf{u}$, is as follows:

$$
\begin{aligned}
& \mathbf{E}=-\alpha_{0} s(\omega) \mathbf{u}, \\
& \alpha_{0}=\frac{\omega_{\mathrm{L}}^{2} \bar{M}}{V_{0}}\left(\frac{1}{\varepsilon_{\infty}}-\frac{1}{\varepsilon_{\mathrm{s}}}\right), \\
& s(\omega)=\frac{\omega^{2}-\omega_{\mathrm{T}}^{2}}{\omega_{\mathrm{L}}^{2}-\omega_{\mathrm{T}}^{2}} .
\end{aligned}
$$

Here, $\bar{M}$ is the reduced mass, $V_{0}$ is the volume of the unit cell, $\varepsilon_{\infty}, \varepsilon_{s}$ are the high-frequence and static permittivities, and $s(\omega)$ is the field factor, which, unlike the case for the LO component, reduces from unity by dispersion.

For $q=0$, the dielectric function vanishes for both LO and IF components. As a consequence, the electric field at the interface is zero when $\mathbf{u}=0$, and 
there is no leakage of fields from the well to barrier and vice versa. A significant consequence of dispersion is the generation of fields at the interface, even though $\mathbf{u}=0$, because of the difference of the dielectric function and field factor between the LO and IF components. This difference introduces two aspects of the LC model regarding the interaction with electrons - LO-like and IF-like. The LO-like interaction occurs at all frequences determined by dispersion; the IF-like interaction occurs only at the frequence at which the sum of the permittivities of the well and barrier equals zero, which occurs typically at a large wave vector.

Without dispersion, the TO component could not exist. The TO dispersion in the quadratic approximation is

$$
\omega^{2}=\omega_{\mathrm{T}}^{2}-v_{\mathrm{T}}^{2} q^{2} .
$$

Coherence with the LO mode is obtained at the wave vector given by

$$
\begin{aligned}
& \omega^{2}=\omega_{\mathrm{T}}^{2}-v_{\mathrm{T}}^{2}\left(q_{\mathrm{T}}^{2}+q_{x}^{2}\right)=\omega_{\mathrm{L}}^{2}-v_{\mathrm{L}}^{2} q^{2}, \\
& q_{\mathrm{T} z}^{2}=-\frac{1}{v_{\mathrm{T}}^{2}}\left[\omega_{\mathrm{L}}^{2}-\omega_{\mathrm{T}}^{2}-v_{\mathrm{L}}^{2} q_{z}^{2}-\left(v_{\mathrm{L}}^{2}-v_{\mathrm{T}}^{2}\right) q_{x}^{2}\right]=-\eta^{2} .
\end{aligned}
$$

The $z$ component must be imaginary and, typically, very large. The TO component is therefore evanescent and severely confined to the interface. As it has no electrical activity, and away from the surface its amplitude becomes infinitesimal, its action in the mechanical boundary condition is simply to ensure that $u_{x}=0$ - its mechanical energy can be ignored. In this case the effective boundary condition reduces to $u_{z}=0$. This provides a useful simplification. Nevertheless, dispersion complicates the calculation of the electron scattering rate in calling for numerical analysis. This is one reason why simpler models - the 3DP and DC - retain popularity.

\section{Coupled modes and hot phonons}

However, those simple models are inadequate for treating the important practical cases where high electron densities and high electric fields introduce the phenomena of coupled plasmon-phonon modes and hot phonons. Plasmon coupling introduces extra dispersion, and high fields produce hot electrons, which, in turn, forces the phonon population to deviate from thermodynamic equilibrium. Any description of these effects will have to take into account variations depending on the frequence and wave vector, and this precludes simple models and calls for the LC approach.

\section{Conclusions}

Where coupled-mode, hot-phonon and other phenomena dependent on special frequence or wave vector are absent, simpler models can be very useful, not only for obtaining approximate estimates for scattering rate, but for suggesting new effects. An example was the speculation of Požela and his group [15, 16] on the effect of inserting a monolayer into a quantum well, which stimulated considerable theoretical activity, resulting in the refinement of the LC model and the recognition of LO-like and IF-like aspects.

Although the LC model has general application, in practice, it has been used with the assumption that the ionic displacement vanishes at the interface. This assumption becomes questionable in systems such as the $\mathrm{Ga}_{1-x} \mathrm{Al}_{x}$ As nanostructure when $x$ is small. In this case the correct mechanical boundary conditions as given by microscopic models must be used.

\section{Acknowledgements}

The author would like to thank Dr. Angela Dyson for many discussions, also the US Office of Naval Research via Grant supervised by Paul Maki.

\section{References}

[1] M.G. Burt, An exact formulation of the envelope function method for the determination of electronic states in semiconductor microstructures, Semicond. Sci. Technol. 3, 739 (1988).

[2] M.G. Burt, The justification for applying the effective-mass approximation to microstructures, J. Phys. Condens. Matter. 4, 6651 (1992).

[3] B.A. Foreman and B.K. Ridley, in: Proc. ICPS24 CDROM Section V-E3 (1999).

[4] B.K. Ridley,Electronsand Phononsin Semiconductor Multilayers, 2nd ed. (Cambridge University Press, 2009).

[5] F.A. Riddoch and B.K. Ridley, On the scattering of electrons by polar optical phonons in quasi-2D quantum wells, J. Phys. C 16, 6971 (1983).

[6] R. Fuchs and K.L. Kliewer, Optical modes of vibration in an ionic crystal slab, Phys. Rev. 140, A2076 (1965).

[7] M. Babiker, Longitudinal polar optical modes in semiconductor quantum wells, J. Phys. C 19, 683 (1986).

[8] J.E. Zucker, A. Pinczuk, D.S. Chemla, A. Gossard, and W. Wiegman, Optical vibrational modes and electron-phonon interaction in GaAs quantum wells, Phys. Rev. Lett. 53, 1280 (1984). 
[9] H. Akero and T. Ando, Envelope-function formalism for phonons in heterostructures, Phys. Rev. B 40, 2914 (1989).

[10]C. Trallero-Giner, F. García-Moliner, V.R. Velasco, and M. Cardona, Analysis of the phenomenological models for long wavelength polar optical modes in semiconductor layered systems, Phys. Rev. B 45, 11944 (1992).

[11] K.J. Nash, Electron-phonon interactions and lattice dynamics of optic phonons in semiconductor heterostructures, Phys. Rev. B 46, 7723 (1992).

[12] B.K. Ridley, Continuum theory of optical phonon hybrids and their interaction with electrons in a quantum well, Proc. SPIE 1675, 492 (1992).
[13] B.K. Ridley, Electron-hybridon interaction in a quantum well, Phys. Rev. B 47, 4592 (1993).

[14] M.P. Chamberlain, M. Cardona, and B.K. Ridley, Optical modes in GaAs/AlAs superlattices, Phys. Rev. B 48, 14356 (1993).

[15]J. Požela, G. Butkus, and V. Jucienè, Electron-optical phonon scattering rates in 2D structures: effects of independent electron and phonon confinement, Semicond. Sci. Technol. 9, 1480 (1994).

[16]J. Požela, V. Jucienè, and K. Požela, Confined electron-optical phonon scattering rates in $2 \mathrm{D}$ structures containing electron and phonon walls, Semicond. Sci. Technol. 10, 1076 (1995).

\title{
ELEKTRONŲ IR POLINIŲ OPTINIŲ FONONŲ SĄVEIKOS NANODARINYJE MODELIAI
}

\author{
B.K. Ridley
}

Esekso universiteto Kompiuterijos mokslų ir elektroninès inžinerijos fakultetas, Kolčesteris, Jungtinè Karalystė 